\title{
Les phénomènes météorologiques et la météorologie intérieure : pour une correspondance sémantico-linguistique
}

\author{
Cristina Ungureanu*
}

Université de Pitești, Târgu din Vale, Nr. 1, Pitești, Roumanie

\begin{abstract}
Résumé. Cette étude se veut une approche franco-anglo-roumaine pour l'appréhension de la correspondance sémantico-linguistique entre les phénomènes atmosphériques et la météorologie humaine. Inspirée d'un travail remarquable et pionnier qui a été mené sur le territoire roumain et qui traite de la terminologie météorologique des phénomènes atmosphériques, notre étude tend à approfondir le même domaine de la météorologie tout en étendant sa portée linguistique à l'état humain qui mettra en exergue la météorologie du corps humain, de son âme et de son esprit. Les phénomènes atmosphériques tels : la pluie, la neige, la giboulée, la température, le vent, le brouillard, la grêle, les précipitations en général, la lumière du soleil, l'éclair ou le tonnerre, ou bien les astres célestes etc., semblent être des organisateurs des humeurs et des sentiments humains à travers la correspondance lexico-sémantique qui s'établit entre ces phénomènes météorologiques et la météorologie intérieure humaine. De ce fait, nous allons dans cet article questionner le fonctionnement et la signification de cette correspondance sémantico-linguistique à travers les interactions météorologiques affectives caractérisant les états d'âme dans trois langues, à savoir le français, l'anglais et le roumain.
\end{abstract}

\begin{abstract}
Meteorological phenomena and internal meteorology: for a semantic-linguistic correspondence. This study is an Anglo-FrancoRomanian approach and deals with the semantic-linguistic correspondence between atmospheric phenomena and human meteorology. Inspired by the remarkable and pioneering work that has been done on the Romanian territory and which deals with the meteorological terminology of the atmospheric phenomena, our study tends to deepen the same field of meteorology while extending its linguistic scope to the human state which will highlight the meteorology of the human body, its soul and its spirit. Atmospheric phenomena such as: rain, snow, sleet, temperature, wind, fog, precipitation in general, sunlight, air, lightning or thunder, or celestial bodies etc., seem to be the organizers of moods and human feelings through the lexical-semantic correspondence that is established between these meteorological phenomena and human meteorology. Therefore, in this article we will examine the functioning and the meaning of this
\end{abstract}

* cristinaungureanu1976@yahoo.com 
semantic-linguistic correspondence through the meteorological-affective interactions characterizing moods in three languages, namely French, English and Romanian.

\section{Problématique et méthodologie}

\subsection{Introduction}

Cette étude a été inspirée d'un travail remarquable et pionnier sur le territoire roumain et européen, La terminologie météorologique roumaine des phénomènes atmosphériques (scientifique versus populaire). L'ouvrage est le fruit d'un projet ${ }^{2}$ national réalisé dans le cadre de l'Institut de Philologie Roumaine «A. Philippide» (l'Académie Roumaine - la filiale de Iași), une entreprise lexicographique de grande envergure, avec des implications substantielles dans l'organisation des mots de la langue dans des champs sémantiques. Comme fins observateurs et preneurs des vies des mots à leurs mains et à leurs plumes, les spécialistes ont harmonieusement couvert toute la gamme scientifique et populaire d'un mot subissant les affres ou bien les régales des conditions atmosphériques [1]. Notre étude vient, par conséquent, approfondir le même domaine de la météorologie mais tout en étendant sa portée linguistique à l'état humain.

La relation suggestive entre le temps et l'humeur est un sujet coutumier, presque ordinaire dans la biométéorologie populaire. Quand même la vérification expérimentale de cette relation reste difficile et complexe [2]. Les études traitant de l'influence du temps sur les émotions humaines sont relativement nombreuses; mais, celles qui portent sur la correspondance linguistique entre les phénomènes atmosphériques et la météorologie humaine sont, nous semble-t-il, faibles en nombre, voire inexistantes. Ainsi, nous cherchons à nous interroger sur la correspondance linguistique qui existerait entre la sphère émotionnelle humaine et les changements du temps, par exemple un temps ensoleillé, instable, serein, nuageux, chaud et humide, etc.

Sans aucune intervention humaine, la planète Terre regorge de phénomènes exceptionnels et captivants. Quoique tout s'explique aujourd'hui par des approches scientifiques, ces «œuvres d'art» peuvent exister, et ceci même figurativement dans l'esprit humain. La neige, le vent, la pluie et le soleil peuvent façonner l'âme d'une manière ou d'une autre et sont ainsi à l'origine d'un subtil mélange d'émotions et de pensées, des états d'âme qui sont le cœur de la relation humaine avec le monde. Pour le dire autrement, la température, le vent, la lumière du soleil, les précipitations, l'air, le tonnerre, etc. deviennent, le plus souvent inconsciemment, des organisateurs humains, des humeurs et des sentiments. Toutes ces idées nous amènent à questionner le fonctionnement et la signification de la correspondance linguistique entre les phénomènes météorologiques et la météorologie intérieure humaine lors des interactions météorologiques affectives caractérisant nos états d'âme dans trois langues, à savoir le français, l'anglais et le roumain.

\subsection{Hypothèses de travail}

Lier temps et humeur par le linguistique serait à la base d'une correspondance faisant du temps, objet extérieur à l'âme, un moteur dynamique de la météorologie humaine intérieure qui se traduirait par et dans le verbe.

${ }^{2}$ Le projet CNCS:PN-II-ID-PCE-2011-3-0656 


\subsection{Cadre théorique et méthodologique}

Nous n'avons pas trouvé dans la littérature de spécialité roumaine ou encore européenne d'ouvrage qui étudie la correspondance de la terminologie des phénomènes atmosphériques dans l'espace affectif. Pour étudier cet objet nous nous intéressons donc à un ouvrage qui a été réalisé par une équipe de chercheurs roumains de l'Académie de Iași intitulé «Terminologia metereologică românească a fenomenelor atmosferice (științific versus popular $^{3}$ ) ». Nous avons pris, de ce fait, le dictionnaire (élaboré) par cette équipe comme corpus et avons développé notre analyse par l'étude figurative de tous ces phénomènes météorologiques mais avec limitation aux états de l'âme. Nous estimons qu'il est important de conserver la division des phénomènes atmosphériques établie par cette équipe de recherche, vu l'étude minutieuse et interdisciplinaire qu'elle effectuait en collaboration avec un groupe de météorologues. Toutefois, pour analyser ce phénomène de correspondance terminologique, nous n'adoptons qu'une perspective linguistique qui vise la structure des lexèmes étant analysée conformément aux dictionnaires roumains, français, anglais.

Nous avons pris en considération, outre les phénomènes atmosphériques à proprement parler, la catégorie des procès ou conséquences dérivés de ces phénomènes comme la glace, le brouillard, le verglas, etc., et la catégorie des astres qui génèrent ces phénomènes : le soleil, la lune, etc. Nous avons parfois dépassé la limitation psychique en faveur des états physiques mais surtout quand nous avons considéré que les expressions étaient pertinentes pour notre étude.

Nous allons présenter succinctement toutes nos découvertes lexicologiques. Cette présentation fait partie d'une étude plus complexe que nous ne pouvons pas détailler ici faute d'espace et par ailleurs, nous n'avons la prétention non plus d'avoir épuisé tout le lexique météorologique dans les trois langues mentionnées.

Il resterait à envisager les baromètres de l'âme à travers toutes les saisons dans leur voyage universel humain.

\section{Approche et analyse : la météo affective}

Les phénomènes atmosphériques constituent certainement un champ très large et dynamique. Ainsi, et dans le dessein de bien mener notre étude, nous avons divisé ce champ en plusieurs sous-champs lexico-sémantiques. Cette catégorisation s'appuie sur la recherche dictionnairique réalisée dans ce domaine.

\subsection{Le sous-champ lexico-sémantique des précipitations}

Cristina Florescu [3] divise le champ en cinq groupes : 1. «Les éléments atmosphériques sous forme de gouttes d'eau à diamètres variables (pluie) ; 2. Les ensembles de petites gouttes d'eau retrouvées en suspension atmosphérique (brouillard) ; 3. Les hydrométéores sous forme de flocons de neige; 4. Les hydrométéores constitués par des sphérules ou fragments de glace à diamètres variables (grêle) ; Les phénomènes hydrométéorologiques représentés par la chute concomitante des flocons de neige et des gouttes de pluie (giboulée) ».

Nous allons traiter seulement les deux premières catégories faute d'expressions atmosphériques contenant les lexèmes grêle ou giboulée ou bien flocons de neige.

\footnotetext{
${ }^{3} \mathrm{La}$ terminologie météorologique roumaine des phénomènes atmosphériques (scientifique versus populaire)
} 


\subsubsection{PLUIE}

Au champ lexical des précipitations, l'élément prototypique est la pluie. Une analyse sémique de ce lexème « qui est parfois mêlée de neige, de grêlons ou verglaçante » ${ }^{4}$ montre des traits sémiques ordinaires /+ froid/, /+vapeur d'eau/, /+liquide/ étant, en fait, une des formes les plus communes de précipitations sur Terre. Elle prend nombre de formes allant de la pluie légère au déluge, de l'averse à la pluie continue, de fines gouttelettes à de très grosses.

Suite à notre recherche, nous constatons que le lexème pluie reçoit des sens métaphoriques supplémentaires, étant présent dans des symboles artistiques, ethnologiques ou bien encore religieux. L'expression de nature humoristique $A$ fi (sau a sta) ca o curcă (ou ca o găină) plouată $\breve{5}^{5}$ ou ca un câine plouat signifie que quelqu'un est triste ou abattu ou bien d'une manière figurative découragé, à contrecœur, ou sans envie.

Un autre syntagme contenant le même lexème pluie Parcă tot îi ninge și-i plouă $\breve{a}^{7}$, renvoie à une personne sombre et qui n'est jamais contente. Par contre, l'expression familière $A$ se face că plouă $\breve{s}^{8}$ sort de la sphère de la tristesse et nous conduit dans la zone de l'indifférence et de la ruse (la personne prétend ainsi n'avoir pas observé une chose ou une situation insatisfaisante) ou de la naïveté Tomber de la dernière pluie.

Le sens physique du lexème atmosphérique pleuvoir se retrouve seulement dans l'expression îmi plouă în gură ou bien am apă în gură / it makes my mouth water. Il s'agit d'une expression populaire, dont le sens a une corrélation avec l'explication anatomique, renvoyant vers ce qu'on appelle la phase céphalique de la digestion. La phase de digestion commence au niveau mental. Dans le cerveau il y a une phase céphalique de la digestion: quand on pense à la nourriture ou que l'on voit ou que l'on sent quelque chose de bon, tout le système digestif est prêt à faire le travail qui a été génétiquement programmé. Après que le cerveau aura enregistré le stimulus, on observera une sécrétion de la salive.

Un autre syntagme méritant notre attention est Ne pas être tombé (né) de la dernière pluie qui signifie : avoir de l'expérience. Cette expression date du XXe siècle. La « dernière pluie » indique un événement frais et récent en opposition avec une personne plus âgée donc dotée de plus d'expérience dans la vie. L'on sous-entend ainsi qu'il s'agit d'une personne qui n'est pas naïve et qui a une lecture expériencée d'une situation. Par contre, lorsque l'on est ennuyeux comme la pluie, on sort de la sphère positive de la pluie pour entrer dans le coté très ennuyeux et très monotone de celle-ci et précisément parce qu'elle renvoie l'image d'un paysage triste et figé dont l'animation ultime se résume à l'eau qui tombe du ciel.

Faire la pluie et le beau temps nous annonce une météo favorable aléatoirement et le verbe «faire » ayant pour sujet implicite l'actant, qui a le pouvoir de décider positivement ou négativement d'une situation, il marque explicitement que le pouvoir est dans les mains d'une personne ou d'un groupe qui ont une posture supérieure. Cette expression, qui date de 1732, pourrait faire référence aux dieux mythologiques tout-puissants qui avaient la possibilité de maîtriser la pluie et le beau temps. Ils avaient le pouvoir suprême de décider $\mathrm{du}$ temps selon leurs envies. Par extension, celui qui, dans son service ou l'espace dans lequel il a des responsabilités importantes, prend toutes les décisions, oriente toutes les

\footnotetext{
${ }^{4}$ https://www.atilf.fr/tlfi, consulté le 13 septembre 2017

${ }^{5} \mathrm{C}$ 'est un adjectif

${ }^{6}$ Etre (ou rester debout) comme une dinde (ou comme une poule) mouillée ou comme un chien mouillé

${ }^{7}$ Il neige et pleut toujours chez lui

${ }^{8}$ Faire mine de rien / la sourde oreille; regarder en l'air; répondre par des pirouettes

${ }^{9}$ Il me vient l'eau à la bouche ; j'en raffole ; j'ai l'eau à la bouche
} 
carrières, punit ou récompense ses collaborateurs, donc celui qui y a tous les pouvoirs ou presque, est aussi celui qui y fait la pluie et le beau temps. ${ }^{10}$

Très intéressant est le syntagme anglais to be (as) right as rain, suggérant figurativement être sain surtout après une maladie ou une expérience négative, en comprenant ainsi que les caractéristiques physiques de la pluie deviennent des caractéristiques morales. La pluie peut même nous sauver dans une situation, par conséquent un britannique peut dire Take a rain check. On dit cette expression I'll take a rain check ou I'll get a rain check lorsqu'on ne peut pas accepter une invitation de quelqu'un pour faire quelque chose mais qu'on voudrait le faire plus tard, à un autre moment (report dans le temps). En français on dirait « Remettons ça à une prochaine fois ». Par exemple, on peut dire qu'on ne peut pas accepter une invitation à dîner ce samedi et demander si cela serait possible le weekend d'après : "I'm sorry, but I'll have to take a rain check for dinner this Saturday. Would next weekend work for you? ». La traduction littérale est « Je vais prendre un chèque de pluie », ce qui ne veut absolument rien dire en français.

Une nouvelle analyse sémique, cette fois-ci du point de vue métaphorique, de ce lexème dans les trois langues, montre qu'il acquiert une gamme plus riche de sens (ce qui évoque ou inspire la pluie par son aspect, sa couleur, son abondance, sa localisation, son but) : /+ennuyeux/, /+triste/, /+indifférent/, /+pénible/, /+naïf/, /rusé/, /+précis/, /+reporté/, /+sain/, /+ puissant/.

On peut conclure que, le phénomène atmosphérique influence l'esprit humain et oriente par conséquent son état d'âme. L'interaction ainsi produite entre ce phénomène et l'esprit humain se traduit par une correspondance sémantico-linguistique.

\subsubsection{BROUILLARD}

Ce terme entre dans la composition de plusieurs syntagmes liés aux phénomènes atmosphériques, syntagmes qu'on retrouve facilement dans les trois langues mentionnées : ceață la sol / brouillard au sol / ground fog etc.

Suite à l'analyse componentielle du lexème brouillard, nous retenons pour notre étude trois traits sémiques : /+gouttelettes d'eau/, /-lumière/, /-visibilité/ qui se retrouvent aussi dans d'autres syntagmes qui ne relèvent pas de phénomènes atmosphériques à proprement parler. Il est important de souligner que les sèmes qui constituent brouillard contiennent dans les trois langues des connotations négatives que nous allons présenter ci-dessous.

Les expressions mi s-a încețoșat privirea / a vedea ca prin ceață indiquent un trouble de vue, renvoyant au processus de ne plus voir clairement. Par contre, les expressions être dans le brouillard, avoir la tête dans le brouillard ou bien le brouillard mental / to be in the fog, my mind is in a fog now ont une aire sémantique se superposant au phénomène atmosphérique. Il s'agit d'un brouillard métaphorique, prenant plus précisément le sens d'avoir la sensation d'avoir le cerveau «embrumé » ou "dans la ouate». C'est la perception subjective d'une personne de ne pas pouvoir penser aussi clairement qu'à l'habitude, ce qui crée donc une confusion ou une perte de mémoire.

E o nebuloasă în capul meu, m-ai bagat în ceață / nager dans le brouillard renvoient vers la situation d'être perdu, de ne pas savoir comment agir. Pour l'expression émerger $d u$ brouillard, il s'agit plutôt ici de sortir d'une période d'incertitude ou de doute. On trouve en français une locution qu'on ne trouve pas dans les autres langues: foncer dans le brouillard qui signale le fait d'agir de manière déterminée, brutale, sans bien connaître la situation, avancer sans se préoccuper de rien ou bien une locution en roumain, inexistante dans les

${ }^{10} \mathrm{http}: / /$ www.expressio.fr/expressions/faire-la-pluie-et-le-beau-temps.php, consultée le 20 septembre 2017 
autres langues a avea ceață în creieri $^{11}$ qui se dit d'une personne qui a consommé de l'alcool ou bien encore un syntagme en anglais où le substantif devient verbe : to fog the $i_{\text {issue }}{ }^{12}$, rendre un problème plus difficile, compliquer les choses.

Sur le plan sémantico-linguistique, on constate qu'il y a une vraie correspondance entre le brouillard physique et le brouillard métaphorique qui, par le verbe, dénote la météo interne du locuteur et traduit une situation de l'instant tout en partant de l'état d'âme.

\subsection{Le sous-champ lexico-sémantique des dépôts}

Les dépôts sont enregistrés autant dans le langage scientifique que dans le langage populaire. Nous nous limiterons ici à indiquer les termes les plus importants qui font partie de cette catégorie : glace, neige, brume, verglas, avalanche, gelée blanche, rosée, glaçons etc.

Les sous-divisions de ce groupe se constituent autour de deux éléments prototypiques la neige et la glace. Ces phénomènes atmosphériques sont liés à l'hiver et par conséquent à la froideur et aux affres de cette saison. On s'attend, par conséquent, à trouver ces sèmes dans la correspondance des sentiments humains. Si sur le plan physique, il s'agit de solidifier un liquide, de sorte qu'il passe à l'état de glace, sur le plan humain et ceci par métaphore une froideur terrible peut aussi glacer les cœurs c'est-à-dire pénétrer le cœur d'un froid très vif. S'il gèle à pierre fendre / de ingheață pietrele, par extension les âmes peuvent aussi se pétrifier à cause d'un manque de chaleur ou bien sous l'effet d'un sentiment violent de peur, d'horreur etc.

En roumain, nous n'avons trouvé le lexème neige dans aucune expression idiomatique et aucun syntagme pour la météo humaine. On ne le trouve en français que dans l'expression blanc comme neige qui montre l'innocence d'une personne, image suggérée en roumain par curat ca lacrima 'propre / immaculé comme la larme'. Une personne qui este de gheața ${ }^{13}$ est indifférente ou émane une sensation désagréable de froideur, causée par la peur, l'émotion etc. A fi cu gheața (sau cu frica) în spate signifie être toujours dans un état d'inquiétude [4].

Etre / rester de glace en français, toujours comme en roumain signifie ne pas se laisser envahir par les émotions. On parle d'un cœur, un visage de glace, un accueil de glace. Toujours dans la même aire sémantique nous signalons les verbes des substantifs mentionnés : Cet examinateur glace les candidats ici avec le sens d'intimider ou bien a-i ingheta cuiva inima (sângele în vine) / glacer le sang de quelqu'un (dans les veines) / turn somebody's blood to ice, glacer quelqu'un d'effroi, de terreur avec le sens figuré de déconcerter, ou bien allant jusqu'à paralyser dans son sens figuré.

Ce sens figuré de «causer une émotion si forte que le mouvement du sang en est comme suspendu » peut être trouvé dans plusieurs expressions dans la langue courante, mais aussi dans des œuvres littéraires. En plus, nous observons que les trois langues font toutes appel à un verbe ou à un substantif (glace, glacer / gheață, a îngheța / ice, to ice) pour créer le monde intérieur des dépôts. Un glaçon peut toujours figurativement se transformer dans une personne froide (glaciale, glacée) et indifférente : Quel glaçon celle-là ! / Un bloc de gheață !

Quand la chaleur apparait ou s'installe, il y a oameni / suflete dezghețate c'est-à-dire des gens ouverts et détendus, qui sont devenus vifs et audacieux tout en échappant de leur maladresse ou timidité [5]. La glace se dégèle, les cœurs se dégèlent aussi, si on dégèle quelqu'un on lui fait perdre sa froideur, sa réserve.

\footnotetext{
${ }^{11}$ Avoir du brouillard dans le cerveau (trad. Lit.)

12 ex. I think we're just fogging the issue by looking at all details (Longman 1995 : 544).

${ }^{13}$ de glace
} 


\subsection{Le sous-champ lexico-sémantique des phénomènes lumineux, sonores, électriques}

Au champ lexical des phénomènes acoustiques, l'élément prototypique est le tonnerre (bruit de la foudre accompagnant l'éclair). En roumain, il est dominé par la terminologie populaire et le syntagme le plus connu transféré au plan émotionnel est a tunat şi a fulgerat $^{14}$ en se rapportant à une personne dont les propos sont empreints d'une forme de violence verbale. L'effet du tonnerre renvoie parfois vers le verbe $a$ trăsni (traquer). Il est intéressant ici de mentionner les correspondances établies à partir de ce verbe dans l'esprit humain. Observons toute la gamme d'états qu'il suggère en roumain:

A rămâne, a se opri (ca) trăsnit (din senin) = a rămâne încremenit, a încremeni (de uimire, de emoție, de groază etc. $)^{15}[6]$

A-i trăsni cuiva ceva (prin cap) sau (rar, tranzitiv) a-l trăsni (pe cineva) prin minte = a-i veni (cuiva) o idee neaşteptată, ciudată, nesăbuită ${ }^{16}$ [7]

A pierde facultatea de a judeca normal; a-și ieși din minți; a se sminti; a se țicni; a înnebuni; a se aliena; a se scrânti ${ }^{17}$ (devenir fou) [8]

A (se) ameți de băutură, a (se) îmbăta ${ }^{18}$.

Ce qui est remarquable est que le tonnerre acquiert un sème positif en français et aussi en roumain dans des expressions familières (une fille du tonnerre, fata e trăsnet etc.); il s'agit d'un superlatif exprimant l'admiration totale, se traduisant par formidable, terrible.

Face like thunder, as black as thunder, son of thunder, blood and thunder sont des expressions idiomatiques qui renvoient aux sèmes atmosphériques de /bruit/, /fort/, /agressivité/, /hostilité/.

Le champ lexical électrique est dominé par le terme 'foudre' (décharge électrique qui se produit par temps d'orage entre deux nuages ou entre un nuage et le sol avec une lumière et une détonation), celui-ci entrant dans beaucoup de syntagmes spécifiques et liés au langage météorologique. Par rapport à sa correspondance dans le plan humain on trouve des syntagmes comme avoir un coup de foudre, manifestation subite de l'amour dès la première rencontre ou bien au pluriel, foudres, avec le sens de reproches. Le verbe foudroyer est lui aussi utilisé figurativement par analogie avec le phénomène physique, dans des syntagmes comme " une crise cardiaque l'a foudroyé » ou "foudroyé quelqu'un du regard ». " Il est parti comme un éclair » ou "un éclair de malice » renvoient toujours au même aspect métaphorique. En roumain, fulger et ses dérivés a fulgera, fulgerător, străfulgera etc. font partie des syntagmes exprimant des émotions: " $\mathrm{m}$-a fulgerat cu privirea ${ }^{19}$ ", «i-au străfulgerat ochii ${ }^{20} »$. La terminologie populaire est elle aussi très suggestive, incluant des termes comme descărcare (décharge), străfulgerare (flash), luminătură (éclair), licărire (lueur), a licări (scintiller) etc. On les retrouve fréquemment dans des phrases comme «El a avut o licărire în ochi când a auzit vestea $»^{21}$ ou "S-a descărcat pe mine când a venit acasă $»^{22}$.

En anglais la série des correspondants émotionnels de lightening semble plus réduite : lightening glance, like lithning, to lighten up.

Un autre phénomène météorologique lumineux est l'arc-en-ciel (curcubeu / rainbow) en forme d'arc, offrant toutes les couleurs du prisme et produit par la réfraction des rayons

\footnotetext{
14 il a tonné et a flashé (trad. Lit.)

15 être pétrifié d'émotion, d'horreur,

16 avoir une idée inattendue, étrange et imprudente

17 devenir fou

18 être ivre

${ }^{19} \mathrm{Il}$ m'a foudroyé du regard

${ }^{20}$ Ses yeux étaient dans un flash

${ }^{21}$ Il a eu une lueur dans ses yeux quand il a entendu les nouvelles.

${ }^{22}$ Quand il est rentré à la maison il s'est énervé contre moi.
} 
du soleil dans les gouttes de pluie, un des spectacles les plus extraordinaires offerts par la nature. On ne le trouve pas dans aucune expression roumaine, française ou anglaise qui suggère une émotion, même s'il est bien connu pour la richesse de ses syntagmes scientifiques et populaires.

\subsection{Le sous-champ lexico-sémantique des astres célestes (étoile, soleil, lune, ciel (comme somme des astres)}

Les astres célestes ne peuvent pas exister sans la sphère céleste. Pourrait-on parler des phénomènes atmosphériques sans parler des astres? Le ciel entre dans la composition de plusieurs syntagmes sous la forme d'expressions figées qu'on retrouve dans plusieurs langues. On est tenté de voir si le Ciel de la Terre devient aussi synonyme avec le Ciel de l'âme. L'expression idiomatique a fi în al 9 lea cer en roumain avec un correspondant direct en français être au septième ciel est en ce sens révélatrice de bonheur infini et de plénitude. En anglais, on retrouve la même aire sémantique dans les expressions: to be in the seventh heaven, to be on cloud nine, to tread on / upon air. Cette locution est ancienne et renvoie à l'idée d'être proche de Dieu. Cependant, les cultures de ces peuples voient un peu différemment ce rapprochement ( 7 versus 9). Les connaissances de l'antiquité reposaient sur des considérations religieuses. Ainsi, le philosophe Aristote imaginait le ciel comme un cristal structuré sur plusieurs niveaux. D'autres théoriciens sont allés encore plus loin. Au XIIIe siècle, le roi Alfonso X de Castille, qui s'intéressait à l'astronomie, démontrait qu'il y avait 127 cieux, tandis que le théologien de Tomasso d'Aquino, qui a vécu au même siècle, a soutenu l'existence de sept cieux.

Avec l'établissement du nombre de cieux, une hiérarchie a été établie entre eux, en réservant les positions ci-dessus aux «bénis ». Ainsi, dire être au septième ciel renvoie vers un sens de plénitude, d'être heureux et bien dans son cœur. En fait, le roumain a aussi d'autres moyens d'exprimer cet état d'âme « am o stare cereasca » (j'éprouve un état paradisiaque).

Un autre astre qui trouve une correspondance dans l'état humain est l'étoile. L'étoile de l'âme a son propre étincèlement, annonçant plusieurs directions :

$A$ vedea stele verzi $i^{23}$, lorsque quelqu'un reçoit un coup violent et ne voit plus la couleur réelle des objets à cause de la douleur; A crede în steaua sa / être confiant dans, en son étoile suggère le fait d'être optimiste et de croire en son destin ; A se naște sub o stea norocoasă (sau rea) / être né sous une bonne (une mauvaise) étoile renvoie à l'idée que cet axe exerce une influence sur la destinée de quelqu'un; A fi cu stea în frunte ${ }^{24}$ être ou se croire meilleur que les autres ; Vai de steana mea (sau a ta, a lui etc.) c'est une interjection comme une sorte de complainte sur son propre destin malheureux.

Toutes ces expressions contiennent dans leur aire sémantique le sème /+lumière/ et /+destin/, connoté ou positivement ou négativement.

Tous les phénomènes météorologiques sont liés principalement à l'énergie solaire, le soleil en étant, la source la plus importante de la planète : « toate procesele de natură fizică sau biologică care au loc pe suprafața Pământului sau în atmosferă sunt generate de energia solară ${ }^{25}$ » [9]. Les sèmes inventoriés de cet astre comme /+chaleur/, /+ rondeur/, /+rougeur/, /+unicité/ se retrouvent aussi dans plusieurs expressions : A se uita (la cineva) ca la soare, regarder quelqu'un avec beaucoup d'amour ou bien la soare te poti uita dar la dânsa $b a^{26}$, suggérant une beauté exceptionnelle d'une personne, idée retrouvée aussi dans ( $A$ fi) rupt

\footnotetext{
${ }^{23}$ Voir des étoiles vertes

${ }^{24}$ Avoir une étoile sur son front (traduction littérale)

${ }^{25}$ Tous les processus de nature physique ou biologique se produisant sur la surface de la Terre ou dans l'atmosphère sont générés par l'énergie solaire.

${ }^{26}$ On peut regarder le soleil mais toi, on ne peut pas te regarder!
} 
din soare c'est-à-dire d'une beauté particulière. Maintes fois on observe que cet astre est personnifié, par exemple dans l'expression esti raza mea de soare / tu es mon rayon de soleil ou rasare soarele pe strada mea / le soleil se lève dans ma rue, en attendant dans mon cœur un meilleur destin.

A la différence du soleil, la lune fait son apparition dans plusieurs syntagmes. Nous allons en mentionner quelques uns et proposer une analyse comparative.

A trăi în lună sau a fi căzut din lună / être dans la lune, c'est-à-dire être distrait, perdu dans ses pensées; A apuca luna cu dinții sau a prinde (sau a atinge) luna cu mâna / to reach for the moon réaliser quelque chose de presque impossible ; A promite (și) luna de pe cer / promise somebody the moon promettre des choses qu'on ne peut pas réaliser; A cere (și) luna de pe cer / ask for the moon demander l'impossible ; Câte-n lună și-n stele (sau în soare $)=$ tout ce qu'on peut imaginer ; A lătra la luna / aboyer à la lune / bark at the moon signifie crier inutilement contre quelqu'un. L'expression provient d'une croyance populaire. Selon celle-ci, les chiens aboient contre la lune (inutilement), car son éclat les blesse.

On trouve en anglais des expressions idiomatiques intéressantes sans correspondance en français ou en roumain mais avec une correspondance directe dans l'esprit humain : to be over the moon être très heureux ou to think one hung the moon lorsque l'on considère quelqu'un comme extraordinaire, le meilleur ou exceptionnellement merveilleux ou bien moon devient verbe comme dans moon about someone or something s'attrister / s'affliger de quelque chose. Le français aussi connait quelques syntagmes inconnus dans les autres langues : être bien luné (être de bonne humeur) ou être mal luné (être de mauvaise humeur) ou to tomber de la lune $e^{27}$ être surpris par le surgissement d'un événement.

La conclusion commune que l'on peut tirer de trois cultures différentes et que ce satellite naturel de la terre reste fortement associé au rêve, voire à la distraction.

\subsection{Le sous-champ lexico-sémantique des rayonnements}

Un rayonnement, synonyme de radiation, désigne le processus d'émission ou de propagation d'énergie et de quantité de mouvement impliquant une onde, une particule ${ }^{28}$.

Manea [10] étudie cette terminologie et explique qu'il y a plusieurs termes / syntagmes scientifiques qui sont liés à la propagation des rayonnements, de la lumière, ou de la chaleur (réfraction, diffusion, effet de serre, flux de chaleur, réflexion diffuse etc.), des notions sur la lumière diffusée, reflétée (crépuscule, lueur, rayon), des notions utilisées pour surprendre l'état de l'atmosphère, du temps clair et du ciel sans nuage (s'éclaircir, serein, éclaircissement etc.). Nous élargissons dans notre étude cette sphère cosmique et nous cherchons les rayonnements humains tout autour de 4 termes essentiels : la lumière, la chaleur, le rayon et le rayonnement.

La lumière avec tous ses résultats - la sérénité, la luminosité et l'éclaircissement (que l'on retrouve dans l'atmosphère clair de lune, lumière des étoiles, lumière du jour, lumière $d u$ soleil, etc.) - sont des constantes présentes dans les trois langues dans plusieurs expressions ou syntagmes tous ayant des rapports avec l'état humain: a i se limpezi sufletul / mintea ${ }^{29}$, a se lumina la minte ${ }^{30}$ senin $^{31}$, a avea o față senina ${ }^{32}$, a se însenina ${ }^{33}$, a avea o față luminoasă $\breve{a}^{34}$, en français $s$ 'éclaircir la voix, la gorge, un sourire éclaira son visage, éclairer sur un sujet, éclairer la pensée, visage serein, jugement serein, une lumière

\footnotetext{
${ }^{27}$ En roumain cazut din cer (tombé du ciel)

${ }^{28}$ https://fr.wikipedia.org/wiki/Rayonnement, consulté le 25 septembre 2017

29 Avoir son âme / son esprit éclairci

${ }^{30}$ Avoir sont esprit éclairé

${ }^{31}$ Serein

${ }^{32}$ Avoir un visage serein

${ }^{33}$ Répandre une espèce de lumière sur le visage

${ }^{34}$ Il avait un visage lumineux
} 
(homme de grande intelligence, de grande valeur), faire la lumière, mettre en pleine lumière, l'espoir luisait encore etc. ou en anglais to light / to brighten up, to set light to, in a different light, to see the light, to have light in one's eyes, be all sweetness and light, according to your own lights, his expression brightened, bright and breezy (cheerful and confident), a bright smile etc.

La chaleur (perçue dans différents degrés, modérée, douce ou bien forte) est aussi un élément présent dans les rayonnements physiques mais aussi psychiques. L'absence ou la présence de la chaleur dans l'atmosphère influence tous les phénomènes atmosphériques. Sur le plan humain, généralement celle-ci renvoie à la passion intérieure, à la force des sentiments tout en construisant toute une gamme séduisante (ardeur, exaltation, passion, vivacité) : suflet cald ${ }^{35}$, a lua cu călduri / nădușeala ${ }^{36}$, a incălzi sufletul ${ }^{37}$, m-a luat flama ${ }^{38}$, flacăra iubirii ${ }^{39}$, a se face foc și pară $\breve{a 0}^{40}$ en français accueil chaleureux, ami chaleureux, la chaleur d'une personne, la chaleur de ses convictione, la flamme d'un regard, parler avec flamme, déclarer sa flamme, ça va chauffer, etc. ou en anglais to flame up ${ }^{41}$, a flame of vengeance / desire / passion, take the heat out of the situation, in the heat of the argument /battle/moment etc.

Le rayon et le rayonnement sont aussi des termes pivots bien ancrés dans le langage météorologique. Comment les rayons affectent-ils l'état d'esprit? Quelques éléments de réponse peuvent être ici approchés comme la lumière miraculeuse provenant de la source. On y retrouve des syntagmes ou expressions presque identiques dans les trois langues: $a$ radia de bucurie / rayonner de joie / radiated joy, a radia de fericire / rayonner de bonheur / to radiate happiness, a vedea o raza / voir un rayon / see a ray, raza de speranta / rayon d'espoir / ray of hope, raza de soare / rayon de soleil / ray of sunshine, etc.

\subsection{Le sous-champ lexico-sémantique des nébulosités}

Les nuages sont regroupés dans un spectre très large, étant classifiés dans des familles, genres, variétés ou bien d'autres particularités [11]. Normalement les météorologues établissent la typologie des nuages en fonction de leur hauteur, structure, origine, couleur, lueur des nuages et de la présence d'autres phénomènes optiques ou électriques [12]. Une analyse componentielle fait ressortir du lexème nuage plusieurs traits sémiques dont nous retenons /+ particule d'eau/, /+glace/ /+condensation/, /+nébulosité/, /+obstruction/ etc. des sèmes qui se retrouvent aussi dans d'autres syntagmes métaphoriques.

Les syntagmes scientifiques qui désignent des nébulosités ont des équivalences en français, en anglais et en roumain : barieră noroasă / barrière de nuages / cloud bar ; vârful norilor / sommet d'un nuage / cloud top ; cer acoperit / ciel couvert / overcast sky, văl noros / nappe de nuages / cloud sheet, etc. [13]. Nous allons nous intéresser à présent aux sens du lexème nuage pour voir s'il se trouve aussi dans des syntagmes connotés différemment. En roumain, Trăiește ou $e$ (cu capul) in nori ou bien parc-ar fi căzut din nori, en français Avoir la tête dans les nuages et en anglais have your head in the clouds se dit d'une personne qui semble absente par rapport à tout ce qui se passe autour d'elle, vivant dans un monde à part, dans des rêves, qui ne veut pas tenir compte de la réalité. $\mathrm{Si}$ les nuages constitués des vapeurs d'eau ou de gouttelettes, des cristaux de glace en

\footnotetext{
${ }^{35}$ Ame chaude

${ }^{36}$ Ressentir de la chaleur

${ }^{37}$ Réchauffer l'âme

${ }^{38}$ La flamme m'a prise (traduction littérale) - tomber amoureux

${ }^{39}$ La flamme de l'amour

${ }^{40}$ Se faire du feu et de la poire (traduction littérale) - devenir très fầché / énervé ; il s'agit de termes populaires (feu pour la grosse chaleur)

${ }^{41}$ Devenir plus fort
} 
suspension dans l'atmosphère sont bénéfiques sur la Terre pour générer la pluie, nous remarquons le transfert négatif affectif qu'on trouve dans la littérature :

«Norii apăsători ai electricității mâniei se izbesc de norii puhavi ai electricității oțărârii » ${ }^{42}[14]$.

Il y a aussi le syntagme "nor de tristețe ${ }^{43}$ un mélange de sentiments qui perturbe l'équilibre mental (<lat. nubilum), un trouble, une tristesse que l'on observe sur le visage «un nour de tristeță se răspîndi pe fața lui » ${ }^{44}$ (variation régionale nour). Aussi les syntagmes être sur un nuage / Il vit sur son petit nuage / He is in a small storm nous signalent l'isolement, un monde ailleurs, particulier ou bien Descendre de son nuage. retrouver son sens pratique, ne plus rêver. De même, si quelqu'un vit under a cloud $^{45}$ (He left the company under a cloud) on comprend qu'il est mal jugé par les autres.

Les nuages ne sont pas statiques, ils sont toujours en mouvement et ils génèrent des évaporations, des condensations, des chutes gravitationnelles des gouttes, des fusions des cristaux, des mouvements multidirectionnels etc. Nous pouvons ainsi conclure que les phénomènes des nébulosités sont observables aussi dans le lexique commun de l'esprit et qu'ils indiquent et suggèrent presque la même confusion et désorientation, le même mouvement ou bien isolement. Le nuage inspire symboliquement des traits sémiques bien évidentes dans notre recherche: / +absent/, / troublé/, /jugé/, /+triste/, /isolé/, /-visible/, /+rêveur/.

\section{Conclusion}

La météorologie du corps humain, de l'âme et de l'esprit est en relation avec les phénomènes atmosphériques réels transposés métaphoriquement dans les humeurs et les sentiments humains et ils sont observables sur le plan linguistique. Si certains résultats peuvent nier la relation entre le temps et l'état émotionnel, on ne peut pas nier la vraie correspondance linguistique entre la météo et le climat affectif.

Notre analyse démontre qu'il y a une correspondance réelle, ici-en bas dans la vie terrestre et que l'atmosphère peut être cosmique et affective ou se révèle être parfois une fusion des deux. L'air, la lumière et le son doivent être caractérisés comme des phénomènes atmosphériques importants. Les expressions atmosphériques étudiées ont diagnostiqué de différents états d'humeur, tout en évoquant la normalité, l'euphorie, l'agressivité, l'état asthénique, la tristesse, la joie, le bonheur, le désespoir, etc. « Le monde que nous habitons, loin de se cristalliser en formes fixes et définitives est un monde de devenir, de flux et d'écoulement : c'est un monde météorologique » [15].

\section{Bibliographie}

C. Ungureanu, Compte rendu pour La terminologie météorologique roumaine des phénomènes atmosphériques (scientifique versus populaire), C. Florescu (coord), L. Manea, E. Tamba, A. Bursuc, F.-T. Olariu, M. Patrașcu, L. Apostol, in Vox Romanica, 76, 357-361 (2017)

M. Persinger, Lag responses in mood reports to changes in the weather matrix, Int. J. Biometeor, 19, 108-114 (1975)

C. Florescu, Precipitațiile, Terminologia metereologică românească a fenomenelor atmosferice (științific versus popular), Iași, Editura Universității „Alexandru Ioan Cuza”, 71 - 105 (2015)

Litera Internațional, Noul dicționar explicativ al limbii române (NODEX), București, Litera Internațional (2002)

\footnotetext{
${ }^{42}$ Les nuages puissants de l'électricité de la colère entrent en collision avec les nuages gonflés de l'électricité de l'indignation.

${ }^{43}$ Nuage de tristesse

${ }^{44}$ Un nuage de tristesse se resplendit sur son visage.

45 (informal) people have bad opinion of them because they have done something wrong
} 
Academia Română, Institutul de Lingvistică „Iorgu Iordan”, Micul dicționar academic, ediția a II-a (MDA2), București, Editura Univers Enciclopedic (2010)

Academia Română, Dicționarul explicativ al limbii române (DEX), ediția a II-a revăzută și adăugită, București, Editura Univers Enciclopedic (2009)

Academia Română, Dicționarul explicativ al limbii române (DEX), idem 6

Academia Română, Dicționarul explicativ al limbii române (DEX), idem 6

O. Bogdan, I. Câmpean, Bazele metodologice ale metereologiei, Universitatea creștină „Dimitrie Cantemir", Facultatea de geografia turismului, Sibiu (2006)

L. Manea, Radiațiile, in Terminologia metereologică românească a fenomenelor atmosferice (științific versus popular), Iași, Editura Universității „Alexandru Ioan Cuza” 145-183 (2015)

C. Cărăbuș, Fenomenele luminoase, sonore, electrice și electro-luminoase, in Terminologia metereologică românească a fenomenelor atmosferice (științific versus popular), Iași, Editura Universității „Alexandru Ioan Cuza” 129-144 (2015)

D. Mihăilă, Introducere în meteorologia practică, Suceava, Editura Universității „Ştefan cel Mare” (2010)

C. Cărăbuş, idem 11

N. Steinhardt, Jurnalul Fericirii, Rohia, Editura Mănăstirii Rohia (2005)

T. Ingold, The Life of Lines, London \& New York, Routeledge (2015) 